\title{
The role of COVID-19 prevalence and cancer preparedness on cancer statistics in the Asia-Pacific region
}

\author{
Jason J. Liu ${ }^{1}$ \\ Published online: 24 September 2020 \\ C) Springer Nature Switzerland AG 2020
}

As the number of diagnosed COVID-19 cases recently exceeded 31 million globally [1], the impact of this pandemic on cancer statistics needs to be elucidated. In the Asia-Pacific region, which has an estimated 8.8 million new cancer cases and nearly half of the world's 18.1 million new cancer cases in 2018 [2], diverse levels of COVID-19 prevalence and cancer preparedness in different countries will contribute to a wide variance in the pandemic's effect on cancer incidence and survivorship across the region.

COVID-19 can affect cancer statistics through various mechanisms, and countries with higher COVID-19 prevalence may have greater changes in these statistics. Cancer stage at diagnosis will be affected by screening delays due to the curtailing of cancer service delivery from shutdowns or the diversion of healthcare resources, or by diagnostic delays due to the reduction of physician visits to avoid COVID-19 infection in the hospital setting. Cancer incidence in the long term may be influenced by the multitude of lingering adverse symptoms among COVID-19 survivors, and by the psychological stress experienced by survivors and family members of those infected. Delays in cancer screening and diagnosis will lead to greater risk of earlier mortality among those with undetected cancer. Cancer survivors are also more likely to die from COVID-19 than the general population, because in addition to potentially being immunosuppressed by the cancer and its treatment, they are more likely to be older and obese, as well as have chronic conditions like diabetes, all of which are risk factors of COVID-19 mortality [3, 4].

The level of cancer preparedness can interact with COVID-19 prevalence to affect cancer statistics. A recent report by The Economist Intelligence Unit (EIU) defined cancer preparedness as how well countries are prepared "to achieve major reductions in premature deaths from cancer,

Jason J. Liu

jasonjliu.jjl@gmail.com

1 Institute of Public Health, National Yang-Ming University, Taipei, Taiwan increase cancer survival rates, and improve the quality of life for cancer patients and survivors [5]." The EIU report measured cancer preparedness using indicators from three domains: (1) cancer-related policy and planning, (2) cancerspecific healthcare delivery, and (3) overall health system and governance [5]. Among the ten Asia-Pacific countries studied in the EIU report, the highest to lowest cancer preparedness scores were assigned to Australia, South Korea, Malaysia, Japan, China, Thailand, Indonesia, India, Vietnam, and the Philippines [5]. Among these countries, the current highest to lowest lifetime COVID-19 prevalence are in India, the Philippines, Australia, Indonesia, Japan, South Korea, Malaysia, China, Thailand, and Vietnam [1]. The EIU report was limited by not covering other low COVID-19 prevalence areas like Taiwan and high prevalence areas like the Middle East. Furthermore, countries with less COVID19 testing and reporting transparency are more likely to underestimate COVID-19 prevalence.

Additional data and research are needed to clarify how COVID-19 prevalence affects cancer preparedness and how COVID-19 prevalence and cancer preparedness interact to affect cancer statistics in countries of Asia-Pacific and other parts of the world. Understanding the global impact of the COVID-19 pandemic on the preparedness and statistics for cancer and other diseases will help refine pandemic response guidelines and quantify the full public health burden of this outbreak.

\section{References}

1. WHO Coronavirus Disease (COVID-19) Dashboard. https://covid 19.who.int/. Accessed 22 Sept 2020

2. International Agency for Research on Cancer. https://www.iarc.fr/ wp-content/uploads/2018/09/Globocan_01.jpg. Accessed 22 Sept 2020

3. National Cancer Institute: risk factors for cancer. https://www. cancer.gov/about-cancer/causes-prevention/risk. Accessed 22 Sept 2020 
4. Williamson EJ, Walker AJ, Bhaskaran K et al (2020) Factors associated with COVID-19-related death using OpenSAFELY. Nature 584(7821):430-436

5. Cancer preparedness in Asia-Pacific: progress towards universal cancer control. https://worldcancerinitiative.economist.com/cance r-preparedness-asia-pacific. Accessed 22 Sept 2020
Publisher's Note Springer Nature remains neutral with regard to jurisdictional claims in published maps and institutional affiliations. 